\title{
Ultrastretchable Conductive Elastomers with a Low Percolation Threshold for Printed Soft Electronics
}

\author{
Hongye Sun, Zongyou Han, and Norbert Willenbacher* \\ Institute for Mechanical Process Engineering and Mechanics, Karlsruhe Institute of Technology (KIT), 76131 Karlsruhe, Germany
}

\begin{abstract}
Stretchable conductors are required for next generation soft electronics. Achieving both high electrical conductivity and high stretchability in conductors composed of elastomers and conductive fillers, however, is challenging. Here, a generic, versatile strategy is reported for producing ultrastretchable conductors exhibiting both superior electrical conductivity $\left(>10^{3} \mathrm{~S} / \mathrm{cm}\right)$ and stretchability $(>1600 \%)$. This is achieved by adding small amounts of immiscible secondary fluid into silver (Ag) filled inks. Capillary forces in these ternary systems induce the self assembly of conductive particle networks at a low percolation threshold (6-7 vol \%), cutting silver consumption by more than $2 / 3$ compared to conventional conductive elastomers. Ag filled polydimethylsiloxane exhibits superior cyclic durability sustaining $100 \%$ tensile strain for 1000 cycles with only a minor loss of conductivity. Ag filled thermoplastic polyurethane displays unprecedented reversibility with nonretarded switching from conductive to nonconductive states during repeated stretching up to $200 \%$ strain. Patterned strain sensors and conductive wirings were $3 \mathrm{D}$ printed to demonstrate the technical feasibility.
\end{abstract}

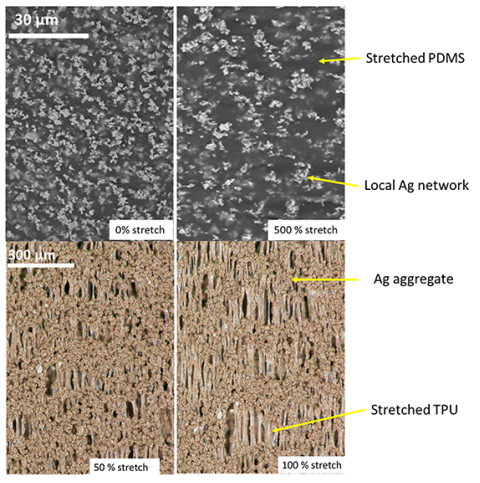

KEYWORDS: conductive elastomers, soft electronics, stretchable sensor, deformable wirings, capillary suspension

\section{INTRODUCTION}

The development of soft electronics with unique flexibility, conformability, and stretchability opens up new frontiers in personalized healthcare, ${ }^{1}$ consumer electronics, ${ }^{2}$ and soft robotics. $^{3}$ Particularly, as the Internet of Things emerges, wearable sensors to collect real time sensing data on human behavior are in high demand. ${ }^{4}$ To integrate components for flexible electronic devices, flexible conductive wiring and printed circuits are critically necessary. Emerging 3D printing tech nologies provide a platform for rapidly prototyping and customizing electronics for individuals. ${ }^{5,6}$ The development of conductive and printable inks for fabricating elastomeric conductors with both high conductivity and stretchability is therefore essential.

Conductive particle filled elastomers are the most commonly used inks for printed stretchable electronics because they are easily adapted to various printing technologies, ${ }^{7-9}$ and formulations can be customized to meet electromechanical and economic specifications. Overcoming the trade off between high electrical conductivity and high stretchability in the current conductive elastomers, however, is a major challenge. While increasing the volume fraction (vol \%) of conductive solids guarantees high conductivity, it deteriorates the stretchability; e.g., increasing the silver $(\mathrm{Ag})$ particle content $\varphi$ from 17 to 22 vol \% in Ag filled polydimethylsiloxane (PDMS) elastomers decreases rupture strain $\varepsilon_{\mathrm{r}}$ from $370 \%$ to $240 \%{ }^{6}$ Rupture may even occur before the loss of electrical conductivity. ${ }^{8}$ This dilemma severely limits the use of polymer based conductive inks in soft electronics. Recent research focused on the use of liquid metal such as eutectic gallium-indium (EGaIn) ${ }^{10}$ or nanoparticles ${ }^{11}$ as electrical interconnections between large conductive particles to simultaneously achieve high conductiv ity, stretchability, and printability. Despite their costly ingredients and complex fabrication processes, such as delicate temperature and process control, ${ }^{10,11}$ these materials are promising candidates for wiring and electrodes due to the robustness of the anchors (EGaln or nanoparticle), but they are not suitable for sensing applications due to small changes in their conductivity when strain is applied.

This work presents a simple and versatile framework for fabricating highly conductive and stretchable elastomers using two material systems, Ag flake filled curable PDMS (Ag PDMS) and Ag filled thermoplastic polyurethane (Ag TPU), to demonstrate this strategy. The latter turns into an elastomer upon evaporation of the solvent. PDMS and TPU have been selected as polymer matrix because they are already widely used in soft electronics due to their good conformability and stretchability. ${ }^{1}$ They represent two classes of stretchable polymers, namely, thermosetting and thermoplastic elasto mers, ${ }^{12}$ thus demonstrating the versatility of our concept. These inks can be printed into sensors as well as conductive wiring, exhibiting a superior combination of electrical conductivity and stretchability. Simply by adjusting the amount of Ag flakes, the conductivity of our materials can be tuned without affecting the stretchability. The initial electrical conductivity $\left(\mathrm{EC}_{0}\right)$ of the elastomer conductors reaches $\sim 10^{3} \mathrm{~S} / \mathrm{cm}$. For Ag TPU, a strain 

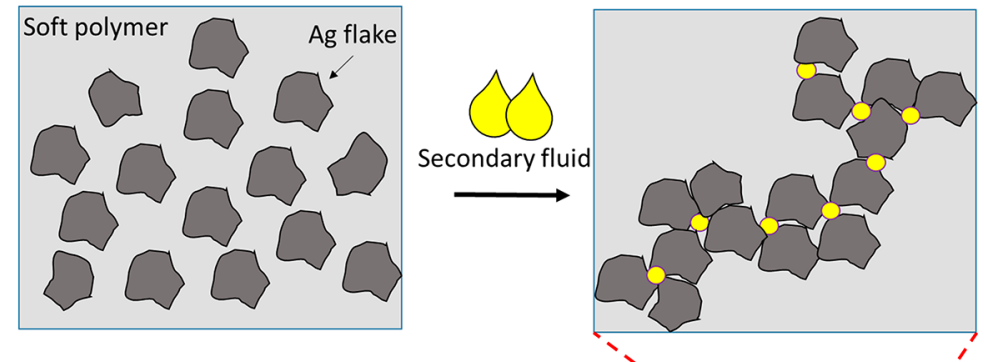

b

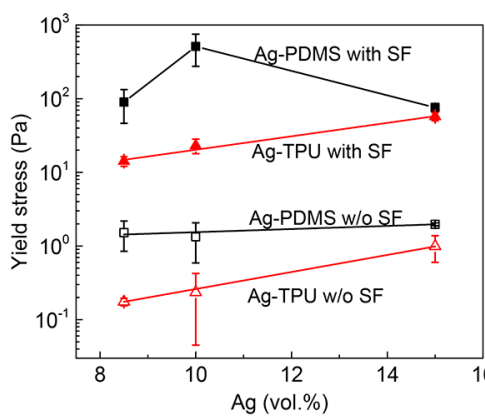

C

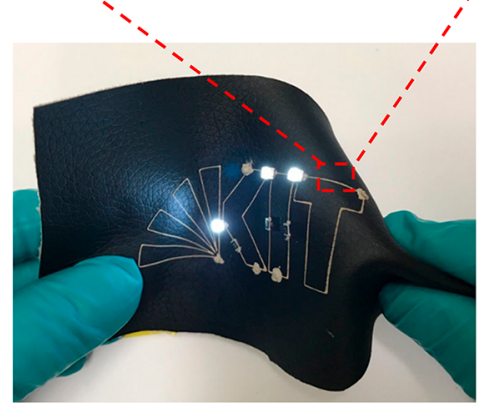

d
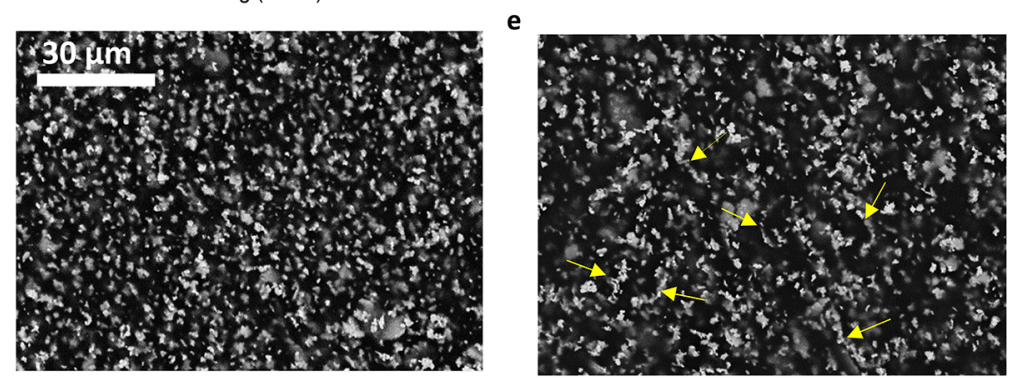

f

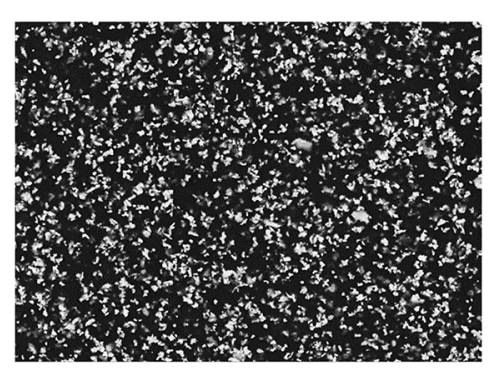

g

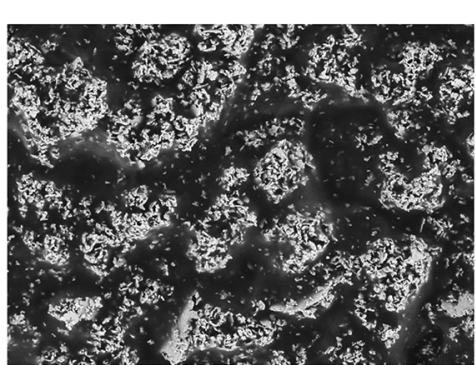

Figure 1. Capillary force induced Ag network built up in PDMS and TPU elastomers upon addition of a secondary fluid. (a) Schematic of network formation. (b) Yield stress of Ag PDMS and Ag TPU with/without secondary fluid as a function of silver flake volume fraction. The secondary fluid concentration was $V_{\mathrm{SF}} / V_{\mathrm{PDMS}}=0.63 \%$ for Ag in PDMS and $V_{\mathrm{SF}} / V_{\mathrm{Ag}}=2 \%$ for Ag in TPU. (c) Conductive network and enhanced yield stress enable the ink to be printed into a conductive circuit (KIT logo) using a 3D printer. (d, e) Surface SEM images of 10 vol \% Ag PDMS elastic conductors without secondary fluid (d) and with secondary fluid (e). Arrows show the particle strings induced by secondary fluid. (f, g) Surface SEM images of 15 vol \% Ag TPU elastic conductors without secondary fluid (f) and with secondary fluid (g). The scale bar shown in (d) is valid for all SEM images.

$\varepsilon$ beyond $1600 \%$ can be applied without mechanical failure. The electrical conductivity (EC) of Ag PDMS conductors remains at $0.15 \mathrm{~S} / \mathrm{cm}$ when stretched to $800 \%$. In addition, Ag PDMS conductors exhibit high cyclic durability; e.g., after 1000 cycles under $100 \%$ tensile strain, the resistance change $\left(R / R_{0}\right)$ at relaxed state is increased by a factor of 2 . Upon stretching, $\mathrm{Ag}$ TPU samples can be repeatedly switched between conductive and nonconductive states without any retardation when subjected to $200 \%$ cyclic strain. Finally, strain sensors and conductive wiring fabricated from both inks using direct ink writing demonstrate practical applications.

The physical mechanism enabling these unique features of our conductive elastomers is the self assembly of the suspended conductive particles induced by the addition of a small amount of a second fluid immiscible with the bulk fluid of the suspension, i.e., the polymer matrix, and the resulting capillary forces in such a ternary solid/fluid/fluid system. ${ }^{13}$ Particles aggregate into clusters even if the secondary fluid does not wet the particles preferentially to minimize the overall free energy of the system. ${ }^{14}$ This yields a particle network with a low percolation threshold, ${ }^{15}$ and high conductivity ${ }^{16,17}$ is achieved with low consumption of the solid conductive particles, ${ }^{18}$ enabling high stretchability without compromising conductivity. The only constraints on the choice of the secondary fluid besides its immiscibility with the bulk phase are that the three phase contact angle $\theta$ of that fluid on the particles within the bulk phase must be $<150^{\circ 19}$ and that it does not disturb the curing reaction. The type and amount of secondary fluid control the morphology of the conductive particle network ${ }^{18}$ and printability (elastic modulus and yield stress) of the paste. ${ }^{20}$ Such so called capillary suspensions comprise a versatile formulation platform for fabricating advanced materials such as highly porous ceramics 
a

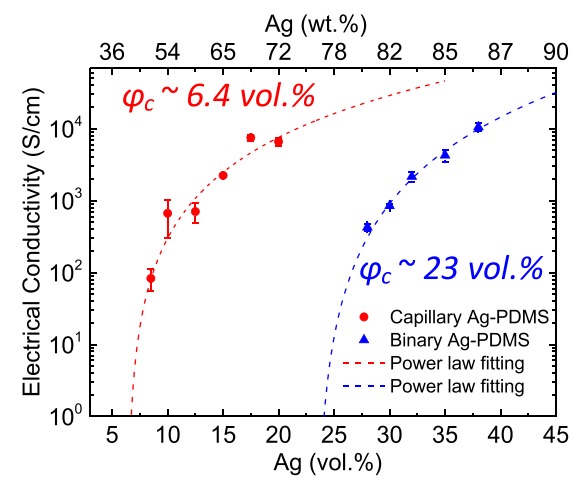

c

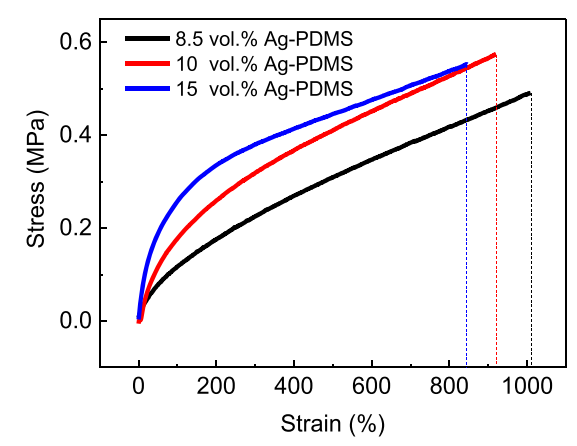

b

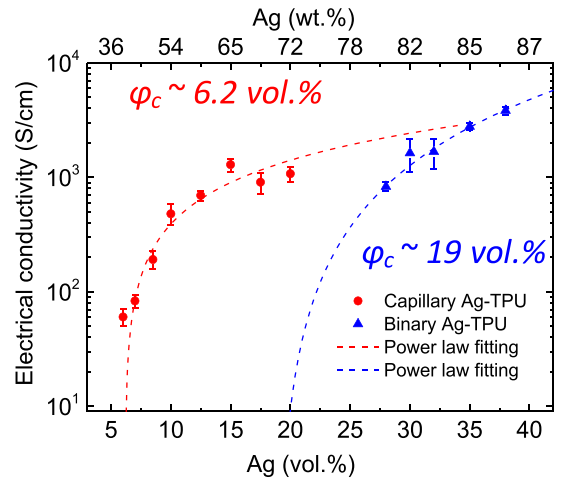

d

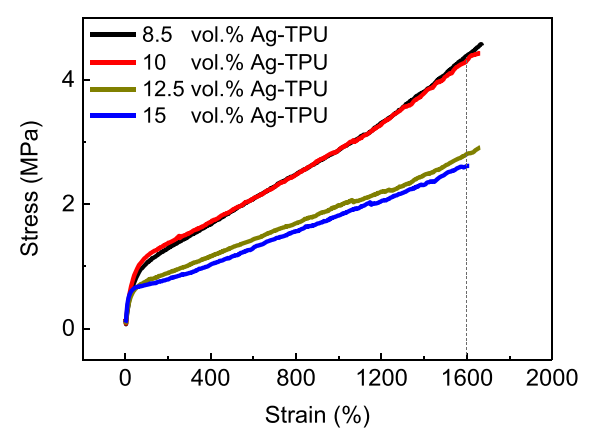

e

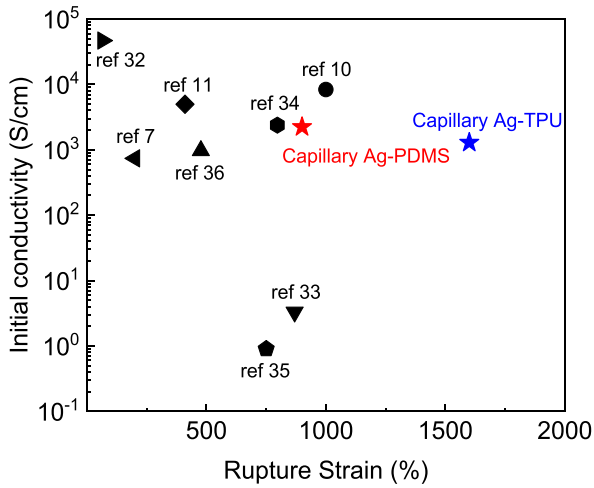

Figure 2. Electrical conductivity and stretchability of capillary elastic conductors. (a) Conductivity of elastic conductor vs volume fraction of Ag flakes for capillary Ag TPU (filled red circle) and binary Ag TPU (filled blue triangle). (b) Conductivity of elastic conductor vs volume fraction of Ag flakes for capillary Ag TPU (filled red circle) and binary Ag TPU (filled blue triangle). (c, d) Stress-strain curves for capillary Ag PDMS (c) and capillary $\mathrm{Ag}$ TPU (d) with different Ag loadings. (e) Comparison of our capillary elastic conductors with the state of the art regarding initial conductivity and maximum stretchability.

with unique strength, ${ }^{21,22}$ high energy density battery electrodes with graded porosity, ${ }^{23}$ or porous silicone. ${ }^{24}$

\section{RESULTS AND DISCUSSION}

Figure la shows a schematic of the capillary bridged Ag network built up by the addition of secondary fluid (SF). Ag flakes self assemble into a $3 \mathrm{D}$ particle network to reduce the overall interfacial energy in the ternary system. ${ }^{18,19}$ Two capillary inks comprising hydrophobic $\mathrm{Ag}$ flakes with an average particle size of $\sim 1 \mu \mathrm{m}$ embedded in two widely used soft elastomers, respectively: PDMS and TPU. Flake shaped Ag was selected because of its large contact area to be bridged by the secondary fluid, ${ }^{25}$ thus providing more transportation pathways for the electrons. The hydrophobicity of the selected Ag flake promises good compatibility with the hydrophobic polymer matrix, which is crucial for good durability of the printed conductors. ${ }^{26}$ The Ag flake morphology and the size distribution are shown in Figure
S1. An ionic liquid (IL, 1 butyl 3 methylimidazolium iodide) and its aqueous solution were used as secondary fluids for Ag TPU and Ag PDMS, respectively. The three phase contact angle $\theta$ of the IL aqueous solution on Ag surrounded by PDMS was $\theta$ $\approx 126^{\circ}$, as determined by the sessile drop method (Figure S2), implying that a capillary state suspension was created. ${ }^{13}$ The secondary fluid concentration for Ag in PDMS was $V_{\mathrm{SF}} / V_{\mathrm{PDMS}}=$ $0.63 \%$ and that for $\mathrm{Ag}$ in TPU was $V_{\mathrm{SF}} / V_{\mathrm{Ag}}=2 \%$. The ionic liquid used in this study contains nitrogen compounds that can cause catalyst poisoning and inhibit PDMS curing; ${ }^{27}$ therefore, an ionic liquid aqueous solution with low nitrogen compound concentration is used as SF, and the addition of SF is regarded to the volume of PDMS matrix. The purpose of adding water is to dilute the concentration of nitrogen compounds in the IL. TPU does not have the catalyst poison issue; thus, pure IL is used as a secondary fluid in this case. The amount of SF in Ag TPU was selected with respect to maximum electrical conductivity 
a

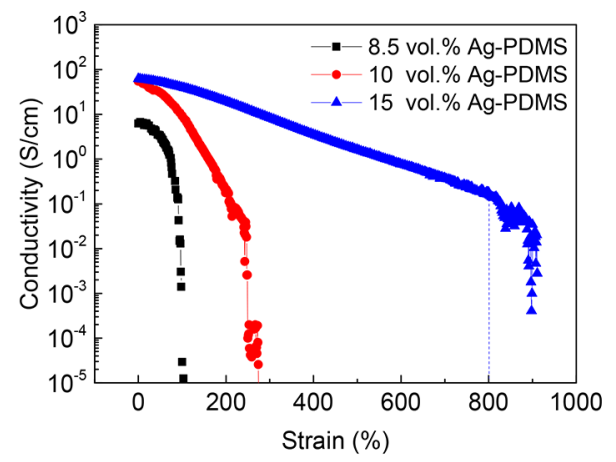

C

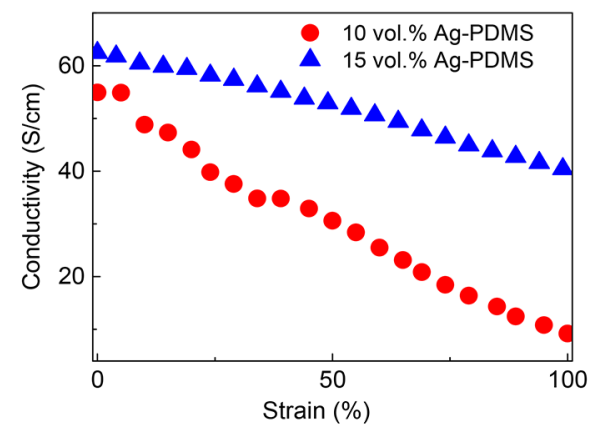

b

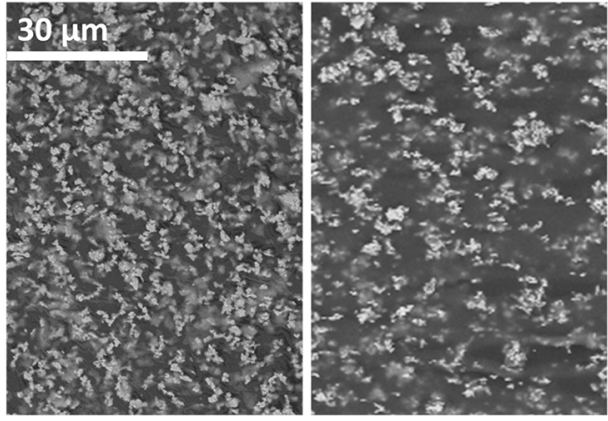

d

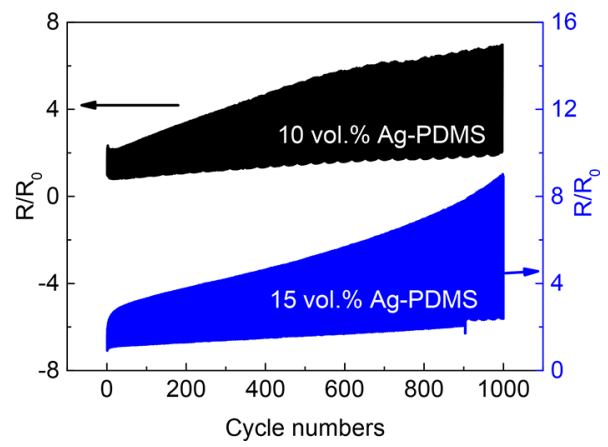

Figure 3. Electromechanical properties of capillary Ag PDMS conductors. (a) Dependence of conductivity on the tensile strain for conductors with different Ag volume fractions. (b) Surface SEM image of $15 \mathrm{vol} \% \mathrm{Ag}$ PDMS unstrained (left) and stretched to 500\% (right), exhibiting a conductivity of $1.6 \mathrm{~S} / \mathrm{cm}$. The scale bar applies to both images. Sample at right was stretched horizontally. (c) Conductivity of 10 and 15 vol \% Ag PDMS as a function of strain $\varepsilon<100 \%$. (d) Cyclic durability of elastic conductors during 1000 cycles at a strain of $100 \%$.

(Figure S3). The yield stress of the inks obtained from rotational rheometry characterizes the strength of the Ag particle network. The data shown in Figure $1 \mathrm{~b}$ confirmed that the yield stress of capillary inks increased by almost 2 orders of magnitude upon addition of SF. This clearly indicates that strong Ag networks are built up in the polymer matrix. The nonlinear increase of yield stress for ternary Ag PDMS results from keeping the ratio of SF to PDMS volume constant in order to guarantee the curability. This results in a decreasing ratio of SF to $\mathrm{Ag}$ and, hence, a drop in yield stress at $15 \mathrm{vol} \% \mathrm{Ag}$ investigated here. The enhanced yield stress of the ternary systems enables ink printability, as demonstrated in Figure 1c where a conductive KIT logo with LEDs was printed using a direct ink writing type $3 \mathrm{D}$ printer. The presence of the $\mathrm{Ag}$ network in the elastomers was further confirmed by scanning electron microscopy (SEM) (Figure 1d$\mathrm{g})$. The Ag flakes were uniformly distributed in the regular binary mixtures with PDMS or TPU (Figure 1d and f). In contrast, particle strings were clearly observed in the capillary Ag PDMS conductor (Figure 1e), as indicated by the arrows. More strikingly, Ag agglomerates were observed in capillary Ag TPU conductors (Figure $1 \mathrm{~g}$ ). The conductivity, however, remained high $\left(>10^{3} \mathrm{~S} / \mathrm{cm}\right)$. The different particle network morphologies observed in capillary Ag PDMS and capillary Ag TPU elastomers are due to the different amounts of secondary fluid. The concentration of SF controls the compactness of particle aggregations, ${ }^{14,28}$ as confirmed by the SEM images of capillary Ag TPU with different SF contents as shown in Figure S4. At lower SF concentration, a particle network structure (Figure S4c) forms similar to that of the Ag PDMS sample shown in Figure 1e; formation of large aggregates occurs at higher SF content, similar to that already observed for capillary model suspensions made from glass beads ${ }^{29}$ or ceramic suspensions used as precursor for porous sintering materials. ${ }^{30}$ For the Ag PDMS system introduced here, catalyst poisoning by the secondary fluid limits the concentration of the latter. Further evaluation of other examples from the large variety of available curable PDMS systems or another choice of SF would be necessary to overcome this limitation.

Parts a and b of Figure 2 show the electrical conductivity vs $\mathrm{Ag}$ particle loading for capillary Ag PDMS and Ag TPU con ductors. The percolation threshold $\varphi_{\mathrm{c}}$ was determined by fitting a power law relationship to these experimental data, ${ }^{31}$ yielding $\varphi_{\mathrm{c}}=6.4 \mathrm{vol} \%$ for capillary Ag PDMS and $\varphi_{\mathrm{c}}=6.2 \mathrm{vol} \%$ for capillary Ag TPU. These elastomeric conductors exhibited high electrical conductivity $\left(\mathrm{EC}>10^{3} \mathrm{~S} / \mathrm{cm}\right)$ for a $\mathrm{Ag}$ concentration of $\varphi=15$ vol \%. Additionally, capillary Ag TPU conductors exhibit a long shelf life, e.g., the EC of specimens did not change substantially even after 6 month storage at room temperature without any encapsulation (see Figure S5). For comparison, the corresponding binary Ag PDMS mixture exhibits $\varphi_{\mathrm{c}}=23 \mathrm{vol} \%$ and binary Ag TPU mixture exhibits $\varphi_{\mathrm{c}}=19 \mathrm{vol} \%$, consistent with the $\varphi_{\mathrm{c}}$ values for $\mathrm{Ag} \mathrm{PDMS}^{6}$ and $\mathrm{Ag} \mathrm{TPU}^{5}$ reported earlier using $\mathrm{Ag}$ flakes similar in size and shape. The drastic reduction in $\varphi_{\mathrm{c}}$ and dramatic improvement in EC result from the self assembly of the Ag particle network induced by capillary forces in the ternary systems. According to the low $\varphi_{\mathcal{c}}$, a high stretchability of capillary ink based elastomeric conductors is anticipated. Respective stress-strain diagrams obtained from tensile testing are shown in parts $\mathrm{c}$ and $\mathrm{d}$ of Figure 2. A rupture strain $\varepsilon_{\mathrm{r}} \approx 900 \%$ is found for all capillary Ag PDMS conductors with Ag loadings between 8.5 and 15 vol \% (see Table S1). The tensile stress during deformation seems to increase slightly with increasing silver loading as expected. It should, however, be kept in mind that the variations in stress-strain curves are almost 
within experimental scatter. For all capillary Ag TPU con ductors, $\varepsilon_{\mathrm{r}}>1600 \%$ is observed. The tensile stress at higher silver content (12.5 and $15 \mathrm{vol} \%)$ in Ag TPU compounds is significantly lower than that at lower silver content ( 8.5 and 10 vol \%); this is attributed to the different particle network structure with more compact aggregates at higher silver loading (see Figure S6). Comparison of rupture strain, electrical conductivity, and percolation threshold is shown in Tables S1-S3. Notably, neither $\varphi_{\mathrm{c}}$ nor conductivity depended much on the elastomer type (see Tables S2 and S3). Rupture strain turned out to be independent of $\mathrm{Ag}$ content in the concentration range relevant for this work (see Table S1), contrary to previous reports showing that stretchability dramatically drops with increasing silver loading. ${ }^{6,8}$ The capillary force driven self assembly of the conductive particle network is not restricted to the particular choice of elastomers and silver particles investigated. This concept should work for other conductive filler/elastomer systems as well.

Figure 2e summarizes the state of the art of stretchability and initial conductivity of stretchable conductors. ${ }^{7,10,11,32-36}$ Elastic conductors from capillary inks deliver a superior combination of good conductivity and high simultaneous stretchability.

Capillary Ag-PDMS Conductors. The electrical conduc tivity of capillary Ag PDMS conductors as a function of tensile strain is shown in Figure 3a. Note, these data were recorded using the two probe method, and thus, the initial conductivity is lower than the values in the percolation curve. The critical strain at which conductivity disappears increases with increased $\mathrm{Ag}$ loading. In particular, capillary Ag PDMS conductors incorpo rating $15 \mathrm{vol} \% \mathrm{Ag}$ flakes exhibited a conductivity of $0.15 \mathrm{~S} / \mathrm{cm}$ at a strain of $800 \%$ without any mechanical failure. This is attributed to the robust Ag network facilitated by the secondary fluid that can withstand high strain without losing conductivity. The microstructures of 15 vol \% Ag PDMS before stretching and at a strain of $500 \%$ are shown in Figure $3 \mathrm{~b}$. Due to the capillary force induced by the secondary fluid, particles still remain in tight contact even at such high strain, which explains that the conductivity is still at a level of $1.6 \mathrm{~S} / \mathrm{cm}$ despite the large deformation. To elucidate the cause for the conductivity loss under strain, Table 1 lists the experimental resistance of 15 vol \% Ag PDMS at 0\% to 500\% strain compared to the change in resistance expected purely form the change in cross section. Geometry corrected resistance values are calculated using the initial resistance measured at $0 \%$ strain and assuming that the conductivity remains the same during deformation and the total

Table 1. Comparison between Experimental Resistance and Calculated Geometry Corrected Resistance of $15 \mathrm{Vol} \% \mathrm{Ag}$ PDMS at 0-500\% Strain $^{a}$

$\begin{array}{cccccc}\text { strain }(\%) & \begin{array}{c}\text { length } \\ (\mathrm{mm})\end{array} & \begin{array}{c}\text { calculated } \\ \text { cross } \\ \left(\mathrm{mm}^{2}\right)\end{array} & \begin{array}{c}\text { experimental } \\ \text { resistance } \\ (\mathrm{ohm})\end{array} & \begin{array}{c}\text { geometry } \\ \text { corrected } \\ \text { resistance } \\ (\mathrm{ohm})\end{array} & \begin{array}{c}\text { experimental } \\ \text { conductivity } \\ (\mathrm{S} / \mathrm{cm})\end{array} \\ 0 & 10 & 1.2 & 1.3 & & 62 \\ 100 & 20 & 0.6 & 2.1 & 5.2 & 40 \\ 200 & 30 & 0.4 & 4.2 & 11.7 & 20 \\ 300 & 40 & 0.3 & 10 & 20.8 & 8.4 \\ 400 & 50 & 0.24 & 23 & 32.5 & 3.6 \\ 500 & 60 & 0.2 & 51 & 46.8 & 1.6\end{array}$

${ }^{a}$ In addition, conductivity data displayed in Figure $3 a$ as calculated from experimental resistance are summarized here. volume of the specimen is constant. As shown in Table 1 , the experimental resistance values are smaller than the resistance calculated from geometrical changes. This must be due to the change of specific resistivity, which is controlled by the structure of the sample spanning network. Thus, we hypothesize that the particles rearrange themselves during stretching up to $300 \%$ strain because the experimental resistance is approximately half as high as expected from geometrical considerations. Therefore, the structural contribution has a positive effect on resistance at small strains, but the network is progressively destroyed at higher strains, resulting in a resistance higher than expected from the cross sectional change at $500 \%$ strain. The higher the $\mathrm{Ag}$ volume fraction, the weaker is the decay of conductivity against strain. In Figure 3c, e.g., the conductivity of 15 vol \% Ag PDMS dropped slightly from 62 to $40 \mathrm{~S} / \mathrm{cm}$ when stretched to $100 \%$. The relative conductivity change $\Delta \mathrm{EC} / \mathrm{EC}$ was 0.55 , where $\mathrm{EC}$ is the conductivity in the stretched state and $\triangle \mathrm{EC}=\mathrm{EC}_{0}-\mathrm{EC}$ is the conductivity change during stretching. The weak depend ence of EC on tensile strain demonstrates the potential of this material to be used as conductive wiring or in flexible circuits. On the other hand, the conductivity of 10 vol \% Ag PDMS decreased from 55 to $9 \mathrm{~S} / \mathrm{cm}$, i.e., $\Delta \mathrm{EC} / \mathrm{EC}=5.1$, indicating the high potential of this class of materials for sensing functions when $\mathrm{Ag}$ loading is only slightly higher than the percolation threshold.

Incorporation of a secondary fluid not only promotes electric interconnections among $\mathrm{Ag}$ flakes but also improves the mechanical bonding between neighboring Ag flakes, resulting in high cyclic durability. This renders the capillary suspension concept a cheap and versatile alternative to incorporating $\mathrm{Ag}$ nanoparticles ${ }^{11}$ or EGaIn particles ${ }^{10}$ to improve the perform ance of elastomer composites. Figure $3 \mathrm{~d}$ shows the cyclic durability of elastic conductors from capillary Ag PDMS undergoing $100 \%$ triangular strain. After a cyclic test comprising 1000 cycles of stretch and release, as shown in Table S4, the relative resistance $\left(R / R_{0}\right)$ in the relaxed, unstrained state increased only by a factor of $\sim 2$, while $R / R_{0}$ at peak strain increased by a factor of 3-4 for both 10 and 15 vol \% Ag PDMS. Figure $\mathrm{S} 7$ shows the resistance change during the first 40 cycles of these two Ag PDMS samples at 100\% strain. Resistance varied in a narrow range always in phase with the applied strain, and no drift was observed during the cycling. Compared with previous reports on elastic conductors, e.g., Ag TPU bearing 1000 cycles under $5 \%$ strain $^{5}$ and $\mathrm{Ag}$ fluorine rubber with $\mathrm{Ag}$ nanoparticles bearing 547 cycles under $50 \%$ strain, ${ }^{11}$ capillary Ag PDMS enduring 1000 cycles at $100 \%$ strain exhibited robust mechanical stability and long term durability, exceeding the current state of the art.

Capillary Ag-TPU Conductors. Electromechanical per formance of capillary $15 \mathrm{vol} \% \mathrm{Ag}$ TPU conductors and a binary 38 vol \% Ag TPU conductor is shown in Figure 4a. When strained to $111 \%$, electrical conductivity of the binary Ag TPU conductor drops to $0.1 \mathrm{~S} / \mathrm{cm}$ and electrical failure occurs at $\sim 125 \%$ strain. The capillary conductor, however, exhibits EC = $0.1 \mathrm{~S} / \mathrm{cm}$ at a strain of $205 \%$ and electrical failure occurs at $\sim 215 \%$. These results clearly demonstrate the benefits of our capillary suspension concept in terms of electrical conductivity under strain combined with a drastically lower silver consumption. Compared to the capillary Ag PDMS conductor where 15 vol \% Ag PDMS maintained a stable conductivity of $0.15 \mathrm{~S} / \mathrm{cm}$ at $800 \%$ strain, the critical strain where $0.1 \mathrm{~S} / \mathrm{cm}$ of $\mathrm{Ag}$ TPU was relatively small, presumably due to the $\mathrm{Ag}$ aggregates forming a conductive network in TPU. The weaker 
a
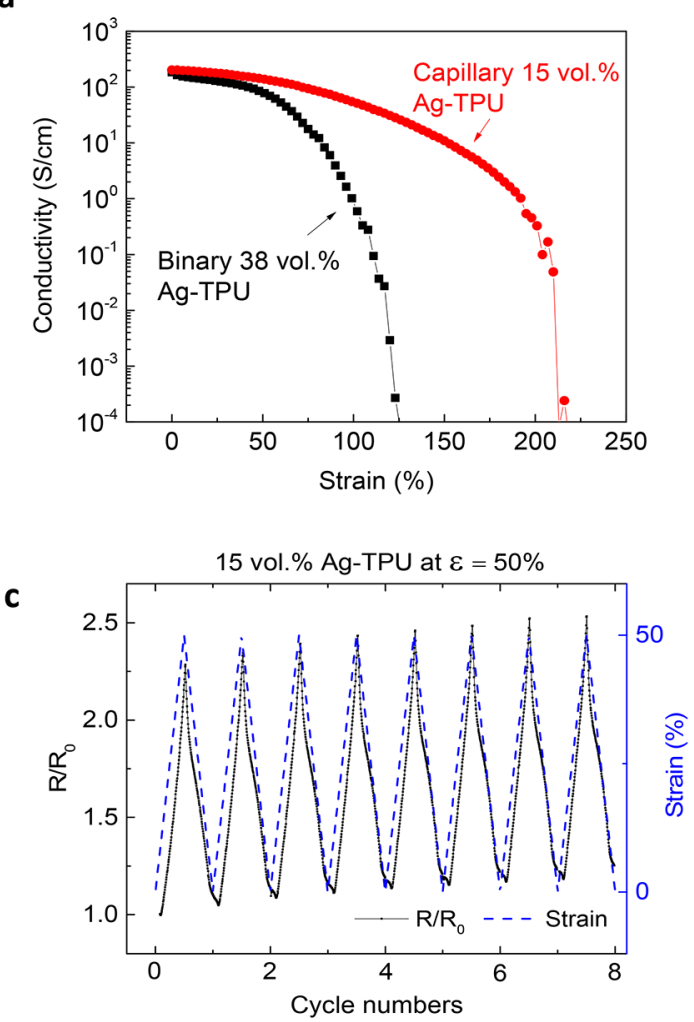

e

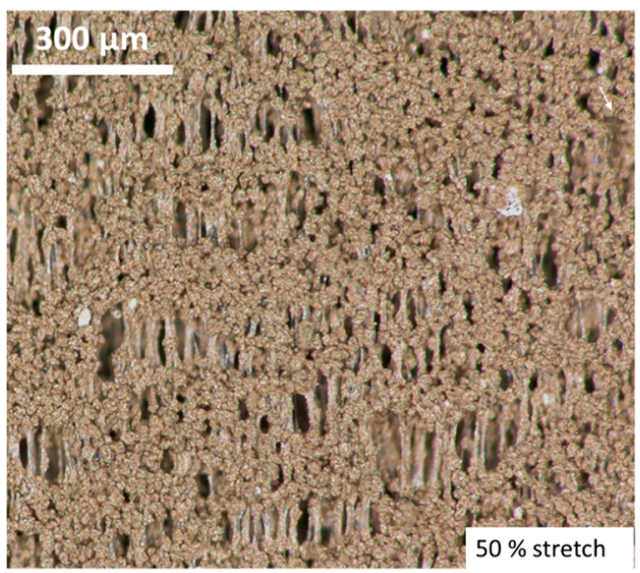

b
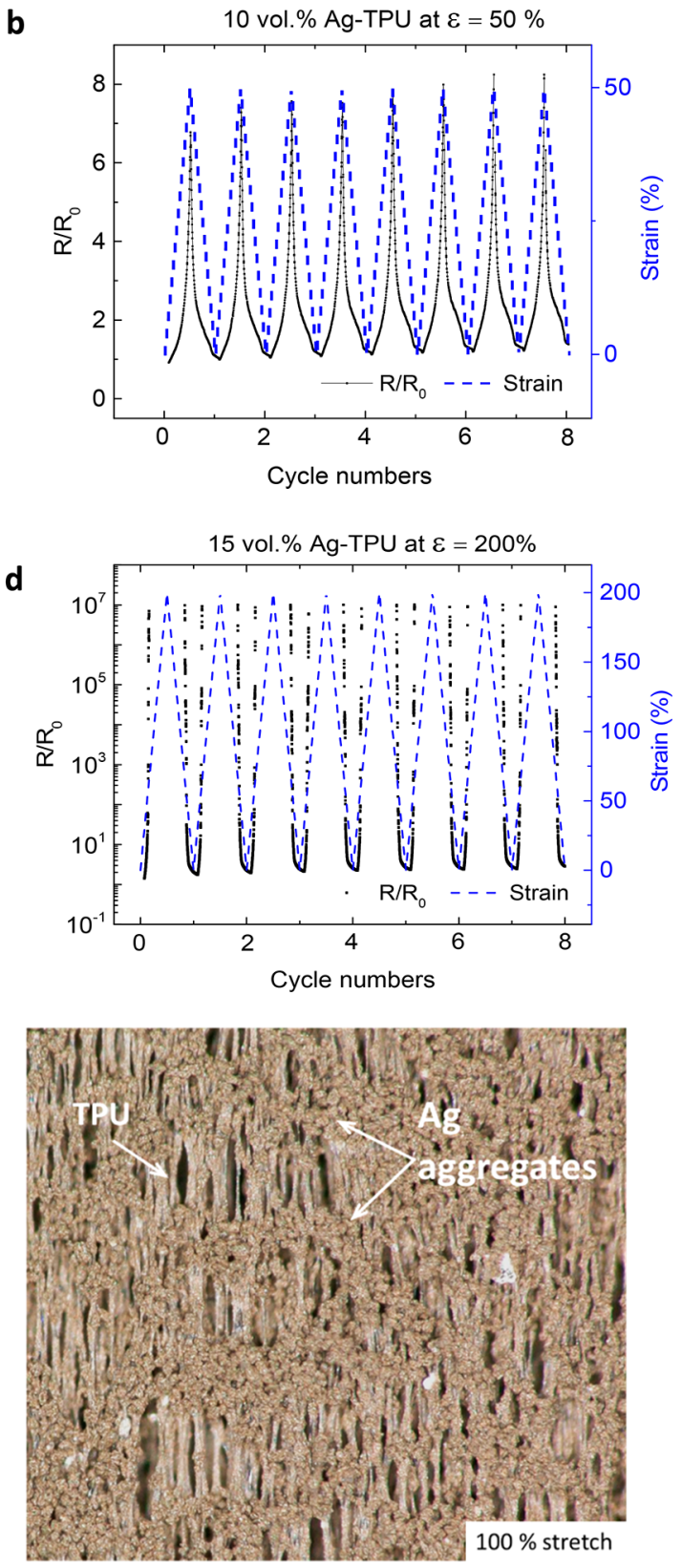

Figure 4. Electromechanical properties of capillary Ag TPU conductors. (a) Dependence of conductivity on the tensile strain for a capillary 15 vol \% $\mathrm{Ag}$ TPU conductor in comparison to a binary $38 \mathrm{vol} \% \mathrm{Ag}$ TPU conductor. (b) Resistance change $R / R_{0}$ of a $10 \mathrm{vol} \% \mathrm{Ag}$ TPU conductor during cyclic tests at a strain of $\varepsilon=50 \%$. (c) Resistance change $R / R_{0}$ of $15 \mathrm{vol} \% \mathrm{Ag}$ TPU at a cyclic strain of $\varepsilon=50 \%$. (d) Resistance change $R / R_{0}$ of $15 \mathrm{vol} \% \mathrm{Ag}$ TPU for 8 cycles at a cyclic strain of $\varepsilon=200 \%(f)$. In (b-d) the resistance change is shown in black, and the applied strain is shown as a blue dotted line. (e) Optical microstructure of capillary $15 \mathrm{vol} \% \mathrm{Ag}$ TPU stretched at 50\% (left) and 100\% (right) strain. The black regions are voids within the sample, and the silver agglomerates and TPU polymer are shown by arrows.

bonds between neighboring aggregates can easily break compared to the Ag strings observed in the PDMS network. This hypothesis is further supported by the performance of 15 vol \% Ag TPU in cyclic strain tests shown in Figure S8. Compared to the superior durability of capillary Ag PDMS for 1000 cycles at a cyclic strain of $100 \%, \mathrm{Ag}$ TPU can only endure a cyclic strain of $50 \%$ for 600 cycles, beyond which the resistance increases abruptly. This is attributed to the different micro structures of both composites: Ag PDMS comprises a capillary network of mostly individual particles, whereas a percolating network of particle aggregates is formed in Ag TPU. The electromechanical performance of capillary conductor with different Ag loadings is shown in Figure S9. The electrical failure strain gradually increases as the silver loading increases, which is consistent with the observation in capillary Ag PDMS conductor.

The sensitivity of 10 and 15 vol \% Ag in TPU against tensile strain was further evaluated during repeated stretching up to $50 \%$ strain in 8 cycles following two initial cycles. As shown in Figure $4 \mathrm{~b}$ and $\mathrm{c}, R / R_{0}$ was $\sim 8$ for $10 \mathrm{vol} \% \mathrm{Ag}$ TPU and $\sim 2.5$ for $15 \mathrm{vol} \% \mathrm{Ag}$ TPU. Consistent with the observation for capillary Ag PDMS conductors, low Ag loading samples are suitable for sensing applications, while high Ag loading samples are suitable for wiring.

To investigate the reversibility, samples of 15 vol \% Ag TPU were subjected to $200 \%$ strain. $R / R_{0}$ measured after two initial 
a

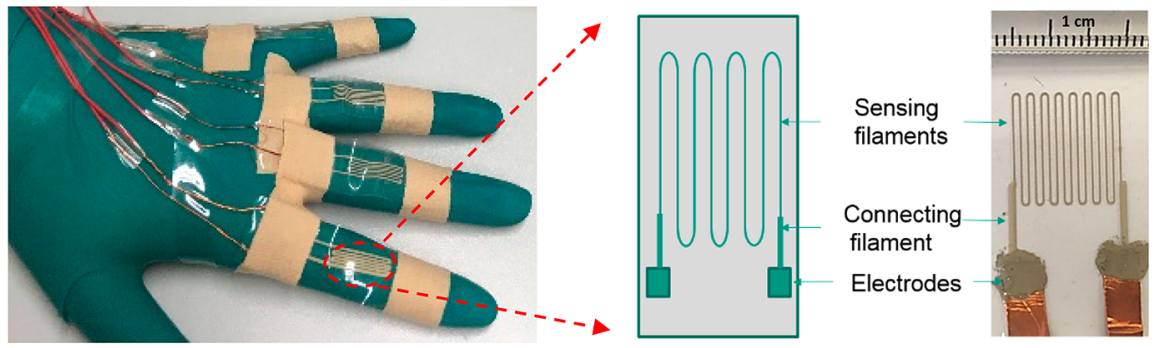

b

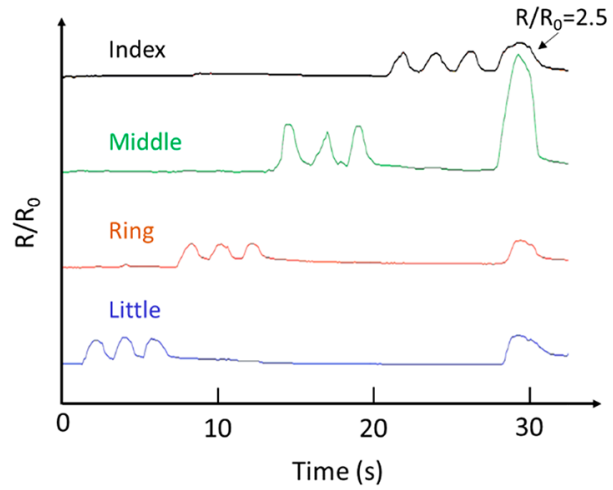

C

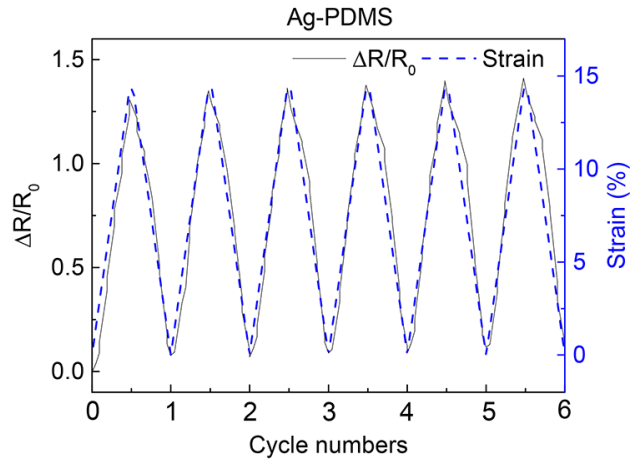

Figure 5. Application of capillary Ag PDMS elastic conductors as strain sensors. (a) Photo of a sensing glove (left). Serpentine design of strain sensor, device scheme (middle), and stretchable sensor prototyped using a 3D printer (right). (b) Relative resistance output of the fingers during hand motion. Each finger bent three times, and four fingers fully bent together afterward. (c) Strain sensor performance of 9 vol \% Ag PDMS during 6 triangular strain cycles at a maximum strain of $15 \%$ recorded as the relative resistance change $\Delta R / R_{0}$ (black line). The applied strain is shown as a blue dotted line.

a

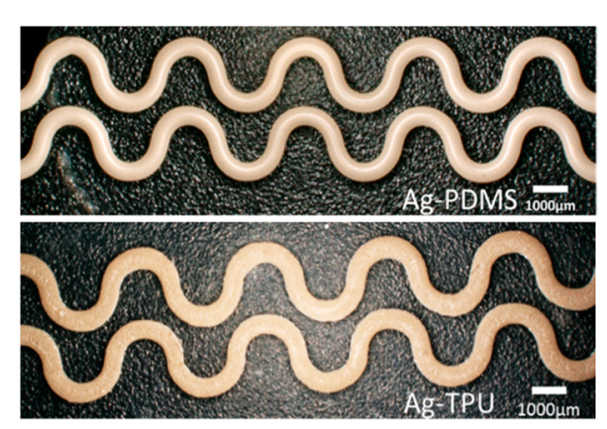

b

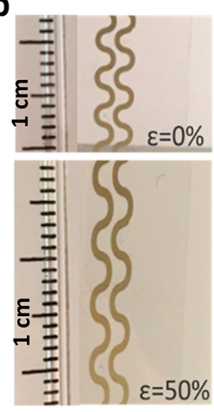

C

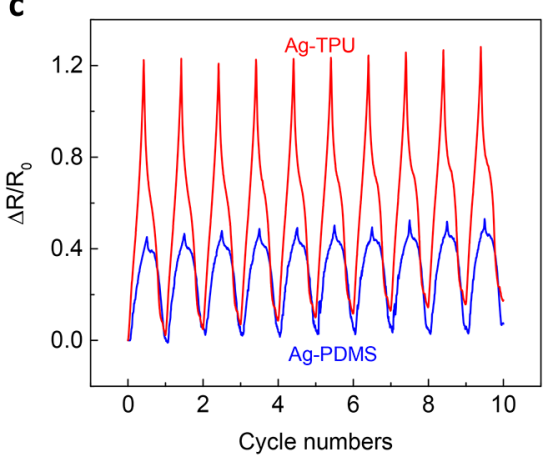

d

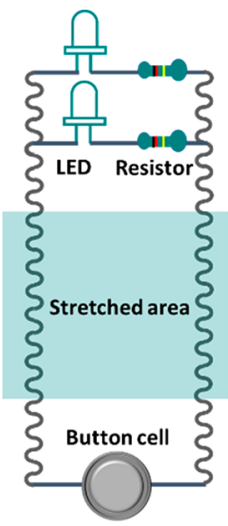

e

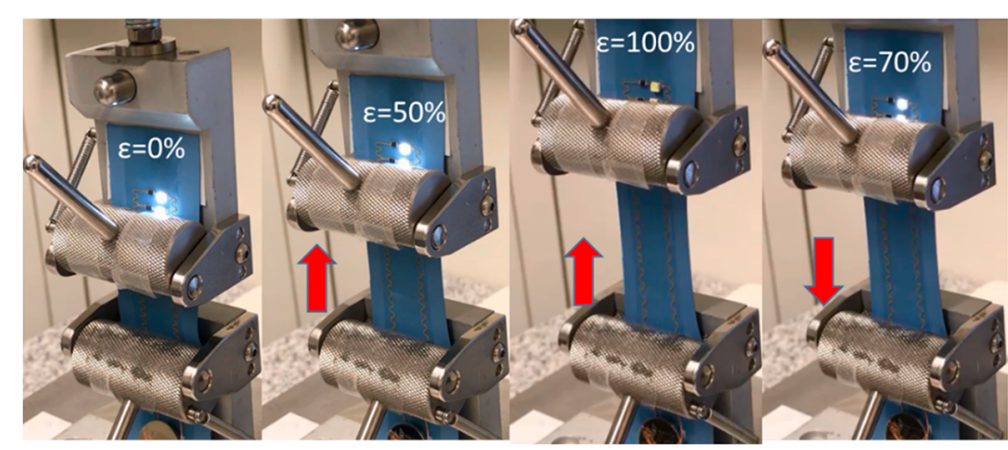

Figure 6. Applications of capillary elastic conductors as conductive wiring. (a) Microscopy images of printed tortuous wires obtained using tapered nozzle with a diameter of $200 \mu \mathrm{m}$ for Ag PDMS and $250 \mu \mathrm{m}$ for Ag TPU. (b) Photographs of Ag PDMS wires in the relaxed state and under 50\% tensile strain. (c) Relative resistance change $\Delta R / R_{0}$ of wirings for 10 triangular strain cycles at a maximum strain of $50 \%$. (d) Stretchable circuit design. (e) Photographs of the circuit printed from $15 \mathrm{vol} \% \mathrm{Ag}$ TPU during stretch and release. 
cycles at cyclic strains of $200 \%$ is shown in Figure 4 d. $R / R_{0}$ was increased by 7 orders of magnitude, and the sample was clearly nonconducive at peak strain. $R / R_{0}$ was always in phase with the applied strain. From one cycle to another, however, the sample switched between conductive and nonconductive states when stretched and released without delay. To the best of our knowledge, such unique reversibility has not been reported before. To further look into the reversibility, the structural evolution during a $100 \%$ strain cycle was recorded using optical microscopy. This peak strain was chosen because the sample is at the transition between conductive and nonconductive, as indicated in Figure $S 10$, where $R / R_{0}$ changes by $>2$ orders of magnitude and its peak varies from one cycle to another. Figure $4 \mathrm{e}$ and Movie S1 show that most of the deformation takes place in silver free regions, i.e., the silver conductive network is hardly affected even at high strain and the loss of conductivity corresponds to a loss of contact among silver particles without changing the overall structure of the network induced by capillary forces. Figure S11 and Movie S2 show that, after the first initiation cycle, the sample relaxed to essentially the same microstructure after every strain cycle, which explains the recovery of conductivity after strain release. This is a unique feature of the capillary network and would not be observed in a binary mixture of polymer and conductive particles with its more uniform particle distribution.

As a proof of concept for capillary suspension based elastic conductors, strain sensors were fabricated from inks of low $\mathrm{Ag}$ loading and conductive wiring was fabricated from inks of high $\mathrm{Ag}$ content by direct ink writing. Figure $5 \mathrm{a}$ shows a glove with four integrated strain sensors, the serpentine design of the strain sensor, and a sensor prototype printed from capillary $9 \mathrm{vol} \% \mathrm{Ag}$ PDMS ink. Copper foils were used as electrodes. The sensors were printed on a substrate made from the same PDMS as used for the conductive ink to avoid delamination. Figure $5 \mathrm{~b}$ shows the performance of the sensors on the glove during finger bending. Each finger was bent 3 times separately, and afterward all fingers were bent simultaneously (see Movie S3). The $R / R_{0}$ signal confirms that the sensors respond to the bending without delay and fully recover after the strain is released. Figures $5 \mathrm{c}$ shows the performance of the sensors made from capillary Ag PDMS. The relative resistance change $\Delta R / R_{0}$ is shown for 6 cycles of triangle strain after two initial cycles. The strain sensor exhibits repeatable and in phase responses to the applied strain. The sensitivity of the sensor was characterized by the gauge factor $(\mathrm{GF})=\left(\Delta R / R_{0}\right) / \varepsilon$. Our sensors show a GF of 9.1 for 9 vol \% Ag PDMS at a strain of $15 \%$. Performance of the sensor made from 10 vol \% Ag TPU ink is shown in Figure S12, and a GF of 7.2 at a strain of $30 \%$ is obtained. These GF values are in the same range as previously reported data for sensors including about twice the amount of silver. ${ }^{5,37}$

An ideal circuit or wiring should maintain low resistance at large deformation. Although capillary conductors exhibit a superior combination of high conductivity and good stretch ability simultaneously, the resistance fluctuation caused by local strain concentration should be minimized through structure design. Inspired by the pioneering work of Rogers et al..$^{38-40}$ to obtain stretchable metal electrodes, tortuous and wave shaped wires were printed onto the same soft polymer substrate by direct ink writing of PDMS and TPU based capillary ink including 15 vol \% Ag. The amplitude and wavelength of the printed feature were $1 \mathrm{~mm}$ and $2 \mathrm{~mm}$, respectively. The wiring was tested under a triangle strain of $50 \%$ for 10 cycles. The microscopy images of patterned wirings before stretching are shown in Figure 6a. Wirings made from Ag PDMS in the relaxed and stretched states are compared in Figure $6 \mathrm{~b}$. Figure $6 \mathrm{c}$ shows the relative resistance change $\Delta R / R_{0}$ over 10 cycles after 2 initial cycles. Wirings printed from both inks had low $\Delta R / R_{0} \approx 1.2$ for $\mathrm{Ag}$ TPU and $\Delta R / R_{0} \approx 0.5$ for Ag PDMS. This is in the same range as recently reported for Ag PDMS, ${ }^{41}$ but in this work it was achieved at a much lower Ag loading ( $15 \mathrm{vol} \%$ Ag compared to, e.g., 30-40 vol \% $\mathrm{Ag}$ in ref 41) and without additional prestraining treatment. To fully demonstrate the wiring capabilities of our conductive elastomers, a printed stretchable circuit made from 15 vol \% Ag TPU combined with LEDs, resistors, and button cell battery is shown in Figure 6d. Further details of the setup can be found in Figure S13. The circuit was stretched to $70 \%$ strain (Movie S4) and 100\% strain (Movie S5) for 3 cycles, respectively. Figure 6e shows photographs of the stretched circuit during its stretch to $100 \%$ strain. It is fully functional at $\varepsilon=50 \%$ and fails at $\varepsilon=100 \%$, as indicated by the on/off of LED lights. However, when strain is released to $70 \%$, the circuit functions again. This demonstrates the outstanding reversibility of the capillary Ag TPU conductor.

\section{CONCLUSION}

In this work a versatile, cost effective route was introduced to fabricate conductive elastomers with simultaneous high conductivity $\left(\mathrm{EC}>10^{3} \mathrm{~S} / \mathrm{cm}\right)$ and stretchability $\left(\varepsilon_{\mathrm{r}}>\right.$ $1600 \%$ ) - at a silver consumption way below the state of the art. Even at low particle loadings, a sample spanning network of conductive particles self assembles in the polymer matrix when a small amount of a second fluid immiscible with the polymer is added. We demonstrated the viability of our concept for two commonly used elastomers, PDMS and TPU, filled with micron sized Ag flakes. Percolation thresholds $\varphi_{\mathrm{c}}$ as low as 6.4 and 6.2 vol \% were achieved, enabling high conductivity at low Ag consumption. Moreover, Ag filled PDMS exhibits superior cyclic durability sustaining 100\% tensile strain for 1000 cycles, and Ag filled TPU displays unprecedented reversibility between conductive and nonconductive states. A strain sensor printed with a PDMS ink including 9 vol \% Ag exhibits a GF of 9.1, and a $\Delta R / R_{0}$ of $\sim 0.5$ was obtained for conductive wiring fabricated from an Ag PDMS ink including 15 vol \% Ag.

This simple and cost effective ink fabrication achieved by merely adding a secondary fluid is highly compatible with current manufacturing processes, allowing for rapid and scalable production. Because of their distinct flow properties, they are ideally suited for robocasting as well as conventional screen printing. The ink formulation concept is not restricted to compounding Ag particles with PDMS or TPU polymer-other conductive particles or other curable polymers may be used as well. Accordingly, this class of materials promises substantial advances in the fields of wearable devices, soft robotics, and deformable electronics.

\section{- EXPERIMENTAL SECTION}

Materials. The cross linkable vinyldimethylsiloxy terminated poly dimethylsiloxane (PDMS) with a kinematic viscosity of $v=10000 \mathrm{cSt}$ (molecular weight $M_{\mathrm{W}}=62700 \mathrm{~g} / \mathrm{mol}$ ) was obtained from ABCR $\mathrm{GmbH}$, Germany. The cross linker trimethylsilyl terminated poly (dimethylsiloxane co methyl hydrosiloxane) (designated as PDMS co PMHS) containing 50\% mol of methylhydrosiloxane and with $v=12$ cSt $\left(M_{\mathrm{W}}=950 \mathrm{~g} / \mathrm{mol}\right)$ was supplied by Sigma Aldrich, Germany. The catalyst tris(dibutylsulfide)rhodium trichloride was obtained from Gelest, Inc., U.S.A.

Thermoplastic polyurethane (TPU) Elastollan 35A was obtained from BASF SE, Germany. The solvents, acetone and $N, N$ 
dimethylformamide (DMF), used for TPU dissolution were supplied by Sigma Aldrich, Germany. Hydrophobic silver (Ag) flakes, 99.95\%, with an average size of $1 \mu \mathrm{m}$ were purchased from Great Wall Precious Metals Co., China. A room temperature ionic liquid (IL), 1 butyl 3 methylimidazolium iodide, was supplied by Sigma Aldrich, Germany.

Fabrication of Capillary Ag-PDMS Ink. Ag flakes were dispersed in PDMS using a planetary mixer (SpeedMixer, Hauschild \& Co. KG, Germany) at $2000 \mathrm{rpm}$ for $5 \mathrm{~min}$. The suspension was further refined using a three roll mill (EXAKT 80E, EXACT Advanced Technologies $\mathrm{GmbH}$, Germany) with a minimum roller gap of $5 \mu \mathrm{m}$. The cross linker and catalyst ( 2 vol \% referring to PDMS base volume, respectively) were then mixed with the suspension in the planetary mixer at $2000 \mathrm{rpm}$ for $2 \mathrm{~min}$. An aqueous IL solution (weight ratio of 2:5 between IL and water) was then added to the Ag PDMS suspension as the secondary fluid phase and dispersed with a planetary mixer at $1700 \mathrm{rpm}$ for $1 \mathrm{~min}$. The volume ratio between the secondary fluid and PDMS was set to $0.63 \%$. A greater amount of the secondary fluid inhibited PDMS curing. Before printing, the ink was evacuated for $10 \mathrm{~min}$ to remove air bubbles. Binary Ag PDMS inks were made in the same process without secondary fluid.

Fabrication of Capillary Ag-TPU Ink. Thermoplastic polyur ethane (TPU) Elastollan 35A (22.5 wt \%, BASF SE, Germany) was dissolved in DMF for $18 \mathrm{~h}$, and the solution was further diluted by acetone at a volume ratio of 4:5 between DMF and acetone. Ag flakes were added to the TPU solution by mixing in the planetary mixer at $2000 \mathrm{rpm}$ for $15 \mathrm{~min}$ total in $5 \mathrm{~min}$ increments, waiting $5 \mathrm{~min}$ between subsequent mixing steps similar to those described in ref 5. IL, the secondary fluid, was added to the Ag TPU suspension and mixed using the planetary mixer at $1700 \mathrm{rpm}$ for $1 \mathrm{~min}$. The volume ratio between secondary fluid and Ag flakes was set to $2 \%$. A further increase of secondary fluid would decrease the conductivity, as shown in Figure S3.

Electrical Conductivity Measurement. Both capillary and binary $\mathrm{Ag}$ PDMS and Ag TPU samples were molded into thin rectangular films (10 cm in length and $1 \mathrm{~cm}$ in width) using a doctor blade. $\mathrm{Ag}$ PDMS films with silver content below 15 vol \% were cured in an oven (Vacutherm Heraeus, Germany) at $130{ }^{\circ} \mathrm{C}$ for $1 \mathrm{~h}$. Capillary as well as binary Ag PDMS with silver loading above 15 vol \% were cured at 200 ${ }^{\circ} \mathrm{C}$ for $2 \mathrm{~h}$. The Ag TPU film, as well as the printed green body, was dried/solidified by removing the solvents. Acetone, which helped to obtain uniform TPU solutions, mostly evaporated during the $\mathrm{Ag}$ dispersing step. Ag TPU ink with DMF as the major solvent was printed using a direct ink writing (DIW) type printer. DMF has a boiling point of $153^{\circ} \mathrm{C}$, which means the evaporation is quite low during the printing process at room temperature. After printing, the green body was first put into a fume hood equipped with air circulation for $4 \mathrm{~h}$ to slowly dry the body. This process aims to avoid air bubbles caused by fast solvent evaporation above the boiling point. Then the sample was transferred into a vacuum oven with a temperature of $80{ }^{\circ} \mathrm{C}$ for another $4 \mathrm{~h}$ to completely remove DMF. The film thickness was determined after solidification using a micrometer caliper. Electrical resistance was determined using a four point probe (RLC 200, Grundig, Germany). Initial electrical conductivity was converted from the resistance and the sample dimensions. The percolation threshold $\varphi_{\mathrm{c}}$ for both Ag PDMS and Ag TPU was determined from fitting a power law equation to the conductivity vs the $\mathrm{Ag}$ content data, ${ }^{31}$

$$
\sigma=\sigma_{0}\left(\varphi-\varphi_{\mathrm{c}}\right)^{s} \text { with } \varphi>\varphi_{\mathrm{c}}
$$

where $\sigma$ is the conductivity of the composites, $\varphi$ is the volume fraction of $\mathrm{Ag}$ particles, $\varphi_{\mathrm{c}}$ is the volume fraction of $\mathrm{Ag}$ particles at the percolation threshold, $s$ is the critical exponent, and $\sigma_{0}$ is a prefactor.

Electromechanical Characterization. For tensile testing, inks were molded in a dog bone geometry (S3A, DIN 53504). After solidifying (as described earlier), samples were tested on a uniaxial tensile testing machine (TA XT Plus Texture Analyzer, Stable Micro Systems, U.K.) and connected to a digital multimeter (Fluke 287, Fluke Corporation, U.S.A.) by copper wires. Tensile tests were conducted at $0.3 \mathrm{~mm} / \mathrm{s}$ for Ag PDMS and $0.1 \mathrm{~mm} / \mathrm{s}$ for Ag TPU, similar to that previously reported. ${ }^{5}$ The electrical conductivity under strain was calculated from the measured resistance and initial geometry. The geometry corrected resistance values given in Table 1 are calculated using $R=\rho \times l / A$, assuming constant volume $V=l \times A$ and specific resistivity $\rho$, where $l$ is the length and $A$ is the cross sectional area. For cyclic strain tests, ink filaments were printed onto pure PDMS and TPU dog bone shaped (S3A, DIN 53504) substrates, respectively, using a 3D printer (Voxel8 Developer's Kit printer, Voxel8, Inc., U.S.A.) equipped with $410 \mu \mathrm{m}$ tapered dispensing tips (EFD). The stretch and release speed was kept to $2 \% / \mathrm{s}$, similar to that previously reported. ${ }^{11}$ We applied $15 \mathrm{vol} \% \mathrm{Ag}$ PDMS and $15 \mathrm{vol} \% \mathrm{Ag}$ TPU to the two ends of the dog bone as electrodes and connected them to the multimeter using copper wires.

3D-Printed Sensors and Wiring. The strain sensor with a serpentine design was printed on pure PDMS and pure TPU substrates for capillary Ag PDMS ink and Ag TPU ink, respectively, via the 3D printer (Voxel8 Developer's Kit printer). A tapered dispensing tip (EFD, Nordson, Germany) and a syringe (3 cc, Nordson, Germany) were mounted on the positioning station, and the extrusion speed was controlled by air pressure. Nozzles that were 200 and $250 \mu \mathrm{m}$ in diameter were used for Ag PDMS and Ag TPU, respectively. The syringe was filled with the capillary ink using a spatula to avoid air bubbles. All printing paths were controlled using G code commands that were generated using the open source software Slic3R. The printed body was solidified as described earlier. To characterize the electrical performance, a copper foil was attached to the electrodes and connected to the multimeter. Sensors were tested at $0.6 \mathrm{~mm} / \mathrm{s}$ for Ag PDMS and $0.3 \mathrm{~mm} / \mathrm{s}$ for Ag TPU. Wirings were tested at $0.3 \mathrm{~mm} / \mathrm{s}$ for Ag PDMS and $0.1 \mathrm{~mm} / \mathrm{s}$ for Ag TPU.

Glove Manufacturing and Testing. A sensing glove was produced by attaching the Ag PDMS strain sensors described earlier to laboratory gloves using tapes. A microcontroller device was programmed using a custom Arduino script to monitor the signal and light emitting diodes (LEDs).

Stretchable Circuit Manufacturing and Testing. The stretch able circuit was fabricated by printing 15 vol $\%$ Ag TPU wires onto a commercial rubber band (Domyos 100 Pilates, Decathlon) using 200 $\mu \mathrm{m}$ nozzle. LEDs (SMD 3528) and resistors (SMD 1206) were mounted onto the circuit using conductive silver epoxy adhesive. A $3 \mathrm{~V}$ button cell was connected to the circuit via copper wires. The circuit was stretched using the tensile testing machine described earlier at a speed of $3 \mathrm{~mm} / \mathrm{s}$.

Rheological Measurement. The yield stress of binary and capillary inks was determined using a controlled stress rheometer (Haake Mars II, Thermo Scientific, Germany) equipped with a vane geometry (Z20 according to DIN 53019 1, $10 \mathrm{~mm}$ vane diameter).

SEM Observation. Ag flake morphology and the particle micronetwork were imaged by scanning electron microscopy (SEM) (S 4500; Hitachi High Technologies Europe GmbH, Krefeld, Germany).

Optical Microscopy. An optical microscope (VHX 950F, Keyence, Germany) was used to image the structural evolution during stretching. A dog bone shaped Ag TPU specimen with $15 \mathrm{vol} \% \mathrm{Ag}$ was stretched from $0 \%$ to $100 \%$ strain and released back to $0 \%$ for 10 cycles at an increment of $10 \%$ strain using a homemade fixture. A red dot was marked to ensure the same location was always observed.

Contact Angle Measurement. The three phase contact angle of secondary fluid on $\mathrm{Ag}$ within the polymer phase was measured using a sessile drop tensiometer (SCA 20, DataPhysics Instruments $\mathrm{GmbH}$, Filderstadt, Germany). Ag flakes were compressed into a pellet shape (25.4 $\mathrm{mm}$ diameter, $7 \mathrm{~mm}$ thickness) using a hand press and immersed in the PDMS base. Secondary fluid was squeezed from a needle (1.2 $\mathrm{mm}$ diameter) hanging above the PDMS and settled down on the Ag pellets. Droplet shape was recorded via the equipped camera, and the three phase contact angle was obtained via image analysis (ImageJ).

\section{ASSOCIATED CONTENT}

Values of rupture strain $\varepsilon_{\mathrm{r}}$, electrical conductivity values, comparison of percolation thresholds $\varphi_{\mathcal{c}}$ changes of $R / R_{0}$ 
over 1000 cycles, morphology of Ag flakes and particle size distribution, three phase contact angles of three liquids on hydrophobic $\mathrm{Ag}$, dependence of electrical conductivity of Ag TPU on the amount of secondary fluid, silver network in $15 \mathrm{vol} \% \mathrm{Ag}$ TPU at different amounts of secondary fluid contents, electrical con ductivity of capillary Ag TPU conductors, silver network comparison between 10 and $15 \mathrm{vol} \% \mathrm{Ag}$ TPU, resistance variations during the first 40 cycles, cyclic durability of 15 vol \% Ag TPU for 860 cycles, electromechanical proper ties of capillary Ag TPU conductors, resistance change $R /$ $R_{0}$ of $15 \mathrm{vol} \% \mathrm{Ag}$ TPU for 8 cycles, optical microstructure images of capillary $15 \mathrm{vol} \% \mathrm{Ag}$ TPU prior to deformation, strain sensor performance of 10 vol \% Ag TPU for 6 triangular strain cycles, and setup of stretchable circuit (PDF)

Structural evolution of capillary 15 vol \% Ag TPU during stretch and release between $0 \%$ and $100 \%$ strain with steps of $10 \%$ strain (AVI)

Microstructures of capillary $15 \mathrm{vol} \%$ Ag TPU at $0 \%$ strain state from cycle 0 to cycle 10 (AVI)

Prototype of a data glove for finger motion motoring (MP4)

Stretchable circuit stretched to $70 \%$ strain for 3 cycles at a speed of $3 \mathrm{~mm} / \mathrm{s}$ (MP4)

Stretchable circuit stretched to $100 \%$ strain for 3 cycles at a speed of $3 \mathrm{~mm} / \mathrm{s}$ (MP4)

\section{Notes}

The authors declare the following competing financial interest(s): The design and material fabrication methods have been submitted for pending European patents.

\section{ACKNOWLEDGMENTS}

This work was financially supported by the German Research Foundation (Grant no. WI 3138/26 01). We thank BASF SE for their kind support with TPU materials.

\section{REFERENCES}

(1) Kenry; Yeo, J. C.; Lim, C. T. Emerging flexible and wearable physical sensing platforms for healthcare and biomedical applications. Microsystems \& Nanoengineering 2016, 2, 16043.

(2) Amjadi, M.; Kyung, K. U.; Park, I.; Sitti, M. Stretchable, skin mountable, and wearable strain sensors and their potential applications: a review. Adv. Funct. Mater. 2016, 26 (11), 1678-1698.

(3) Wallin, T.; Pikul, J.; Shepherd, R. 3D printing of soft robotic systems. Nature Reviews Materials 2018, 3, 84-100.

(4) Patel, S.; Park, H.; Bonato, P.; Chan, L.; Rodgers, M. A review of wearable sensors and systems with application in rehabilitation. Journal of neuroengineering and rehabilitation 2012, 9 (1), 21.

(5) Valentine, A. D.; Busbee, T. A.; Boley, J. W.; Raney, J. R.; Chortos, A.; Kotikian, A.; Berrigan, J. D.; Durstock, M. F.; Lewis, J. A. Hybrid 3D printing of soft electronics. Adv. Mater. 2017, 29, 1703817.

(6) Guo, S. Z.; Qiu, K.; Meng, F.; Park, S. H.; McAlpine, M. C. 3D printed stretchable tactile sensors. Adv. Mater. 2017, 29, 1701218.

(7) Matsuhisa, N.; Kaltenbrunner, M.; Yokota, T.; Jinno, H.; Kuribara, K.; Sekitani, T.; Someya, T. Printable elastic conductors with a high conductivity for electronic textile applications. Nat. Commun. 2015, 6, 7461.

(8) Larmagnac, A.; Eggenberger, S.; Janossy, H.; Vörös, J. Stretchable electronics based on Ag PDMS composites. Sci. Rep. 2015, 4, 7254.

(9) Lee, H.; Lee, J.; Seong, B.; Jang, H. S.; Byun, D. Printing Conductive Micro Web Structures via Capillary Transport of Elastomeric Ink for Highly Stretchable Strain Sensors. Advanced Materials Technologies 2018, 3 (2), 1700228.

(10) Wang, J.; Cai, G.; Li, S.; Gao, D.; Xiong, J.; Lee, P. S. Printable Superelastic Conductors with Extreme Stretchability and Robust Cycling Endurance Enabled by Liquid Metal Particles. Adv. Mater. 2018, 30 (16), 1706157.

(11) Matsuhisa, N.; Inoue, D.; Zalar, P.; Jin, H.; Matsuba, Y.; Itoh, A.; Yokota, T.; Hashizume, D.; Someya, T. Printable elastic conductors by in situ formation of silver nanoparticles from silver flakes. Nat. Mater. 2017, $16(8), 834$.

(12) Sastri, V. R. Plastics in medical devices: properties, requirements, and applications; William Andrew: 2013.

(13) Koos, E.; Willenbacher, N. Capillary forces in suspension rheology. Science 2011, 331 (6019), 897-900.

(14) Koos, E. Capillary suspensions: Particle networks formed through the capillary force. Curr. Opin. Colloid Interface Sci. 2014, 19 (6), 575-584.

(15) Zhang, X.; Yuen, M. M. f. Electrical and thermal conductive paste composition and method of reducing percolation threshold and enhancing percolating conductivity using the same. U.S. Patent US9870843B2, 2018

(16) Zhang, X.; Sun, H.; Yang, C.; Zhang, K.; Yuen, M. M.; Yang, S. Highly conductive polymer composites from room temperature ionic liquid cured epoxy resin: effect of interphase layer on percolation conductance. RSC Adv. 2013, 3 (6), 1916-1921.

(17) Amoabeng, D.; Velankar, S. S. A review of conductive polymer composites filled with low melting point metal alloys. Polym. Eng. Sci. 2018, 58 (6), 1010-1019.

(18) Sun, H.; Zhang, X.; Yuen, M. M. Enhanced conductivity induced by attractive capillary force in ternary conductive adhesive. Compos. Sci. Technol. 2016, 137, 109-117.

(19) Koos, E.; Willenbacher, N. Particle configurations and gelation in capillary suspensions. Soft Matter 2012, 8 (14), 3988-3994.

(20) Schneider, M.; Koos, E.; Willenbacher, N. Highly conductive, printable pastes from capillary suspensions. Sci. Rep. 2016, 6, 31367.

(21) Maurath, J.; Willenbacher, N. 3D printing of open porous cellular ceramics with high specific strength. J. Eur. Ceram. Soc. 2017, 37 (15), $4833-4842$.

(22) Dittmann, J.; Maurath, J.; Bitsch, B.; Willenbacher, N. Highly porous materials with unique mechanical properties from smart capillary suspensions. Adv. Mater. 2016, 28 (8), 1689-1696.

(23) Bitsch, B.; Gallasch, T.; Schroeder, M.; Börner, M.; Winter, M.; Willenbacher, N. Capillary suspensions as beneficial formulation concept for high energy density Li ion battery electrodes. J. Power Sources 2016, 328, 114-123.

(24) Roh, S.; Parekh, D. P.; Bharti, B.; Stoyanov, S. D.; Velev, O. D. 3D printing by multiphase silicone/water capillary inks. Adv. Mater. 2017 29 (30), 1701554.

(25) Maurath, J.; Bitsch, B.; Schwegler, Y.; Willenbacher, N. Influence of particle shape on the rheological behavior of three phase non brownian suspensions. Colloids Surf., A 2016, 497, 316-326.

(26) Araki, T.; Nogi, M.; Suganuma, K.; Kogure, M.; Kirihara, O. Printable and stretchable conductive wirings comprising silver flakes and elastomers. IEEE Electron Device Lett. 2011, 32 (10), 1424-1426.

(27) Fehn, A.; Weidinger, J. Use of rhodium crosslinking silicone elastomers for producing baking molds. Google Patents: 2006

(28) Fortini, A. Clustering and gelation of hard spheres induced by the Pickering effect. Phys. Rev. E 2012, 85 (4), 040401.

(29) Bossler, F.; Koos, E. Structure of particle networks in capillary suspensions with wetting and nonwetting fluids. Langmuir 2016, 32 (6), 1489-1501. 
(30) Dittmann, J.; Willenbacher, N. Micro structural investigations and mechanical properties of macro porous ceramic materials from capillary suspensions. J. Am. Ceram. Soc. 2014, 97 (12), 3787-3792.

(31) Toker, D.; Azulay, D.; Shimoni, N.; Balberg, I.; Millo, O. Tunneling and percolation in metal insulator composite materials. Phys. Rev. B: Condens. Matter Mater. Phys. 2003, 68 (4), 041403.

(32) Kim, Y.; Zhu, J.; Yeom, B.; Di Prima, M.; Su, X.; Kim, J. G.; Yoo, S. J.; Uher, C.; Kotov, N. A. Stretchable nanoparticle conductors with self organized conductive pathways. Nature 2013, 500 (7460), 59.

(33) Liu, Z.; Fang, S.; Moura, F. A.; Ding, J.; Jiang, N.; Di, J.; Zhang, M.; Lepró, X.; Galvão, D.; Haines, C.; et al. Hierarchically buckled sheath core fibers for superelastic electronics, sensors, and muscles. Science 2015, 349 (6246), 400-404.

(34) Wang, Y.; Zhu, C.; Pfattner, R.; Yan, H.; Jin, L.; Chen, S.; Molina Lopez, F.; Lissel, F.; Liu, J.; Rabiah, N. I.; et al. A highly stretchable, transparent, and conductive polymer. Science advances 2017, 3 (3), e1602076.

(35) Keplinger, C.; Sun, J. Y.; Foo, C. C.; Rothemund, P.; Whitesides, G. M.; Suo, Z. Stretchable, transparent, ionic conductors. Science 2013, 341 (6149), 984-987.

(36) Liang, J.; Tong, K.; Pei, Q. A water based silver nanowire screen print ink for the fabrication of stretchable conductors and wearable thin film transistors. Adv. Mater. 2016, 28 (28), 5986-5996.

(37) Kim, I.; Woo, K.; Zhong, Z.; Ko, P.; Jang, Y.; Jung, M.; Jo, J.; Kwon, S.; Lee, S. H.; Lee, S.; et al. A photonic sintering derived Ag flake/nanoparticle based highly sensitive stretchable strain sensor for human motion monitoring. Nanoscale 2018, 10 (17), 7890-7897.

(38) Rogers, J. A.; Someya, T.; Huang, Y. Materials and mechanics for stretchable electronics. Science 2010, 327 (5973), 1603-1607.

(39) Lin, H.; Pan, K.; Yang, F.; Qin, Q. Analysis of stress concentration phenomenon in stretchable interconnects. In 2015 International Conference on Mechatronics, Electronic, Industrial and Control Engineering (MEIC 15); Atlantis Press: 2015.

(40) Gonzalez, M.; Axisa, F.; Bulcke, M. V.; Brosteaux, D.; Vandevelde, B.; Vanfleteren, J. Design of metal interconnects for stretchable electronic circuits. Microelectron. Reliab. 2008, 48 (6), 825832.

(41) Li, C. F.; Li, W.; Zhang, H.; Jiu, J.; Yang, Y.; Li, L.; Gao, Y.; Liu, Z. Q.; Suganuma, K. Highly Conductive Ag Paste for Recoverable Wiring and Reliable Bonding Used in Stretchable Electronics. ACS Appl. Mater. Interfaces 2019, 11 (3), 3231-3240. 
Karlsruher Institut für Technologie

\section{Repository KITopen}

Dies ist ein Postprint/begutachtetes Manuskript.

Empfohlene Zitierung:

Sun, H.; Han, Z.; Willenbacher, N.

Ultrastretchable Conductive Elastomers with a Low Percolation Threshold for Printed Soft Electronics.

2019. ACS applied materials \& interfaces, 11

doi: $\underline{10.5445 / / R / 1000099506}$

Zitierung der Originalveröffentlichung:

Sun, H.; Han, Z.; Willenbacher, N.

Ultrastretchable Conductive Elastomers with a Low Percolation Threshold for Printed Soft Electronics.

2019. ACS applied materials \& interfaces, 11 (41), 38092-38102.

doi:10.1021/acsami.9b11071

Lizenzinformationen: KITopen-Lizenz 